Circuits Systems Signal Process

VOL. 20, No. 1, 2001, Pp. 1-21

\title{
FORWARD, BACKWARD, AND SYMMETRIC SOLUTIONS OF DISCRETE ARMA REPRESENTATIONS*
}

\author{
N. P. Karampetakis, ${ }^{1}$ J. Jones, ${ }^{2}$ and S. Antoniou ${ }^{1}$
}

\begin{abstract}
The main objective of this paper is to determine a closed formula for the forward, backward, and symmetric solution of a general discrete-time Autoregressive Moving Average representation. The importance of this formula is that it is easily implemented in a computer algorithm and gives rise to the solution of analysis, synthesis, and design problems.
\end{abstract}

Key words: ARMA representations, solutions, discrete-time systems, forward, backward, symmetric.

\section{Introduction}

Consider a nonhomogeneous system of linear difference and algebraic equations described in matrix form by

$$
A(\sigma) y_{k}=B(\sigma) u_{k},
$$

where $\sigma$ denotes the forward shift operator, i.e., $\sigma^{i} y_{k}=y_{k+i}$,

$$
\begin{aligned}
& A(\sigma)=A_{0}+A_{1} \sigma+\cdots+A_{q} \sigma^{q} \in R[\sigma]^{r \times r}, \quad \operatorname{rank}_{R(\sigma)}=r \\
& B(\sigma)=B_{0}+B_{1} \sigma+\cdots+B_{q} \sigma^{q} \in R[\sigma]^{r \times m},
\end{aligned}
$$

where $A(\sigma)$ is a regular polynomial matrix (i.e., $\operatorname{det} A(\sigma) \neq 0$ for almost every $\sigma), y_{k}: Z^{+} \rightarrow R^{r}$ is the input, and $u_{k}: Z^{+} \rightarrow R^{m}$ is the input of the system. Following the terminology of [16], we call the set of equations (1) an ARMA representation of $\mathcal{B}$, where $\mathcal{B}$ is the solution space of the system defined by

$$
\begin{aligned}
& \text { Editor: } \\
& \text { menuscript and } \\
& \text { tex file differs. } \\
& \text { we followed } \\
& \text { menuscript }
\end{aligned}
$$

$$
\mathcal{B}=\pi_{y}\left(\mathcal{B}_{f}\right)
$$

* Received March 1996; revised January 1998.

${ }^{1}$ Department of Mathematics, Aristotle University of Thessaloniki, Thessaloniki 54006, Greece. E-mail addresses: karampet@ccf.auth.gr and antoniou@ccf.auth.gr

2 Department of Mathematical Sciences, Loughborough University of Technology, Loughborough, Leics, LE11 3TU, England, U.K. E-mail: jonathan.jones@experian.com

First line is differ from menuscript

Author: $\mathbb{Z}$ and $\mathbb{R}$ in menuscript. 
with

$$
\mathcal{B}_{f}:=\left\{\left(\begin{array}{cc}
y_{k} & u_{k}
\end{array}\right): Z^{+} \rightarrow R^{r} \times R^{m} \mid(1) \text { is satisfied } \forall k \in Z^{+}\right\}
$$

and

$$
\pi_{y}: R^{r} \times R^{m} \rightarrow R^{r} \quad \text { is given by } \pi_{y}\left(y_{k} \quad u_{k}\right)=y_{k} .
$$

In the case where $A(\sigma)=\sigma E-A \in R[\sigma]^{r \times r}$ and $B(\sigma)=B \in R^{r \times m}$, then the ARMA representation (1) is the known generalized state space representation, i.e.,

$$
E x_{k+1}=A x_{k}+B u_{k}
$$

whereas in the case where $\operatorname{det}[E] \neq 0,(2)$ is the known state space representation. For a survey of singular systems of the form (2), see [7].

ARMA representations of the form (1) find numerous applications in the analysis of circuits [12], neural networks [2], economics (the Leontieff model, see [9]), and power systems [14].

The solution of the ARMA representation (2) has been calculated by many different techniques ([1], [6], [10], [13], [15]), and among them we distinguish [8] and [11]. This technique gives a solution of the singular system representation in terms of the fundamental matrix $\phi_{k}$ and the backward fundamental matrix $\tau_{k}$ of $(z E-A)^{-1}$. Following similar methods to those of [8] and [11], we produce in Section 3 a closed formula for the forward, backward, and symmetric solutions of the general ARMA representation (1) in terms of the fundamental matrix $H_{k}$ and the backward fundamental matrix $V_{k}$ of $A(s)^{-1}$. A generalized Leverrier technique for computing the forward fundamental matrix is available [3], so that we may assume that this fundamental matrix is given. We shall show in Section 2 that the backward fundamental matrix is the forward fundamental matrix of the dual polynomial matrix $\tilde{A}(\sigma)=A_{0} \sigma^{q}+A_{1} \sigma^{q-1}+\cdots+A_{q}$ of $A(s)$, and thus we may assume that $V_{k}$ is also given. The whole theory is illustrated via an example in Section 4.

\section{Preliminary results}

We are concerned with the discrete-time ARMA representation (1), where $y_{k} \in$ $R^{r}, u_{k} \in R^{m}, k=0,1, \ldots, N-q$. We assume that $A(\sigma)$ is regular, i.e., $\operatorname{det} A(\sigma) \neq 0$ for almost every $s$. Given regularity, the Laurent series expansion about infinity of $A(s)^{-1}$ exists and is given by

$$
A(\sigma)^{-1}=H_{\hat{q}_{r}} \sigma^{\hat{q}_{r}}+H_{\hat{q}_{r}-1} \sigma^{\hat{q}_{r}-1}+\cdots, \quad|\sigma|>\rho>0,
$$

where $\hat{q}_{r}$ is the greatest order of the zeros of $A(\sigma)$ at $\sigma=\infty$ and the sequence $\left\{H_{k}\right\}$ is known as the forward fundamental matrix [3]. The Laurent expansion about zero of $A(s)^{-1}$ exists and is given by

$$
A(\sigma)^{-1}=V_{-\ell} \sigma^{-\ell}+V_{-\ell+1} \sigma^{-\ell+1}+\cdots, \quad|\sigma|<\rho,
$$


where the sequence $\left\{V_{k}\right\}$ is known [8] as the forward fundamental matrix.

The Laurent expansion about zero of $A(s)^{-1}$ given in (4) is related with the Laurent expansion about infinity given in (3) of the inverse of the dual matrix $\tilde{A}(\sigma)=A_{0} \sigma^{q}+A_{1} \sigma^{q-1}+\cdots+A_{q}$ of $A(\sigma)$, as we can see in the following lemma.

Lemma 2.1. Let the Laurent expansion about infinity of $\tilde{A}(\sigma)^{-1}$ be

$$
\tilde{A}(\sigma)^{-1}=\tilde{H}_{f} \sigma^{f}+\tilde{H}_{f-1} \sigma^{f-1}+\cdots+\tilde{H}_{1} \sigma+\tilde{H}_{0}+\tilde{H}_{-1} \sigma^{-1}+\cdots
$$

and (4) is the Laurent expansion about zero of $A(\sigma)^{-1}$. Then

$$
q+f=\ell \text { and } V_{-i}=\tilde{H}_{-\ell+f+i} \quad \text { for } i=\ell, \ell-1, \ldots, 1,0,-1, \ldots
$$

Proof. We have that

$$
\begin{aligned}
A(\sigma) & =\sigma^{q} \tilde{A}\left(\frac{1}{\sigma}\right) \Leftrightarrow A(\sigma)^{-1}=\sigma^{-q} \tilde{A}\left(\frac{1}{\sigma}\right)^{-1} \stackrel{4}{\Leftrightarrow} \\
A(\sigma)^{-1} & =\sigma^{-q}\left[\tilde{H}_{f} \sigma^{-f}+\tilde{H}_{f-1} \sigma^{-f+1}+\cdots\right] \\
& =\tilde{H}_{f} \sigma^{-q-f}+\tilde{H}_{f-1} \sigma^{-q-f+1}+\cdots \\
& \equiv V_{-\ell} \sigma^{-\mu}+V_{-\ell+1} \sigma^{-\mu+1}+\cdots .
\end{aligned}
$$

Author:

$\stackrel{6}{\Leftrightarrow}$ in menuscript.

Equating the coefficients of the powers of $\sigma$ we obtain the proof of Lemma 2.1

A direct result from Lemma 2.1 is that the Leverrier algorithm in [3] may be used for the computation of both the forward and backward fundamental matrices.

An interesting result that connects the solutions of the ARMA representation (1) and the ones of the dual discrete-time ARMA representation

$$
A_{q} \tilde{y}_{k}+A_{q-1} \tilde{y}_{k+1}+\cdots+A_{0} \tilde{y}_{k+q}=B_{q} \tilde{u}_{k}+B_{q-1} \tilde{u}_{k+1}+\cdots+B_{0} \tilde{u}_{k+q}
$$

in the closed interval $[0, N]$ is given by the following theorem.

Theorem 2.2. (a) If $\tilde{y}_{k}$ is a solution of (7) for the nonzero input $\tilde{u}_{k}$, then the sequence $y_{k}=\tilde{y}_{N-k}$ is a solution of the dual equation (1) for the nonzero input $u_{k}=\tilde{u}_{N-k}$.

(b) If $y_{k}$ is a solution of (1) for the nonzero input $u_{k}$, then the sequence $\tilde{y}_{k}=$ $y_{N-k}$ is a solution of the dual equation (7) for the nonzero input $\tilde{u}_{k}=u_{N-k}$.

Proof. (a) Let $\tilde{y}_{k}$ be a solution of 7 . This implies that 7 is satisfied. Now consider equation (1). If we set $y_{k}=\tilde{y}_{N-k}$ and $u_{k}=\tilde{u}_{N-k}$ and take into account that $y_{k+j}=\tilde{y}_{N-(k+j)}, u_{k+j}=\tilde{u}_{N-(k+j)}, j=0,1, \ldots, q$, we have

$$
A(\sigma) \tilde{y}_{N-k}=\sum_{i=0}^{q} A_{i} \tilde{y}_{N-k-i} \stackrel{7}{=} \sum_{i=0}^{q} B_{i} \tilde{u}_{N-k-i} \stackrel{u_{k}=\tilde{u}_{N-k}}{=} B(\sigma) \tilde{u}_{N-k},
$$

which verifies the first part of the theorem. 
4 N. P. KARAMPETAKIS et al.

(b) In the same way we can prove the second part of the theorem.

A direct result from the Theorem 2.2 is that the backward solution of the ARMA representation (1) comes directly from the forward solution of the dual ARMA representation (7).

\section{Solutions of ARMA representations}

There are three different interpretations of equation (1) [8]:

(1) We may consider that the initial conditions $\left\{y_{0}, y_{1}, \ldots, y_{q-1}\right\}$ are given, and that is desired to determine $y_{k}$ in a forward fashion from the input sequence and the previous values of the output.

(2) We may consider that the final conditions $\left\{y_{N}, y_{N-1}, \ldots, y_{N-q+1}\right\}$ are given, and that is desired to determine $y_{k}$ in a backward fashion from the input sequence and the future values of the output.

(3) We may consider 1 as a relationship between the inputs and outputs, e.g., as in economics, and thus no causality is assumed. It is desired to determine $y_{k}$ for the values $k=q, q+1, \ldots, N-q$, in terms of the input sequence and the initial and final conditions. We could call this the symmetric solution of (1).

\subsection{The forward solution of ARMA representations}

Consider the discrete-time ARMA representation (1), where $A(\sigma)$ is regular, i.e., $\operatorname{det} A(\sigma) \neq 0$ and the Laurent series expansion about infinity for the resolvent matrix exists and is given by (3). Then we have the following theorem.

Theorem 3.1. The whole response of the system (1) will be

$$
\begin{aligned}
y_{k}=\left[\begin{array}{llll}
H_{-k-q} & H_{-k-q+1} & \cdots & H_{-k-1}
\end{array}\right]\left[\begin{array}{cccc}
A_{q} & 0 & \cdots & 0 \\
A_{q-1} & A_{q} & \cdots & 0 \\
\vdots & \vdots & \ddots & \vdots \\
A_{1} & A_{2} & \cdots & A_{q}
\end{array}\right]\left[\begin{array}{c}
y_{0} \\
y_{1} \\
\vdots \\
y_{q-1}
\end{array}\right] \\
+\left[\begin{array}{lllllll}
H_{-k} & H_{-k+1} & \cdots & H_{0} & \cdots & H_{\hat{q}_{r}}
\end{array}\right] \\
+ \\
+\left[\begin{array}{ccccccc}
B_{0} & B_{1} & \cdots & B_{q} & 0 & \cdots & 0 \\
0 & B_{0} & B_{1} & \cdots & B_{q} & \cdots & 0 \\
\vdots & \ddots & \ddots & \ddots & \ddots & \ddots & \vdots \\
0 & \cdots & 0 & B_{0} & B_{1} & \cdots & B_{q}
\end{array}\right]\left[\begin{array}{c}
u_{0} \\
u_{1} \\
\vdots \\
u_{k+\hat{q}_{r}+q}
\end{array}\right]
\end{aligned}
$$


or equivalently

$$
y_{k}=\sum_{i=1}^{q} \sum_{j=1}^{q} H_{-k-i} A_{j} y_{j-i}+\sum_{i=0}^{k+\hat{q}_{r}} \sum_{j=0}^{q} H_{-k+i} B_{j} u_{i+j} \quad \text { with } k=q, q+1, \ldots
$$

Proof. Equating the coefficients of the powers of $\sigma$ in the relation $A(\sigma) \times$ $A(\sigma)^{-1}=I_{r}$, we have

$$
\sum_{n=0}^{q} A_{n} H_{i-n}=\delta_{i} I_{r} \quad \text { or } \quad \sum_{n=0}^{q} H_{i-n} A_{n}=\delta_{i} I_{r}
$$

where $\delta_{i}=0$ for $i \neq 0$ and $\delta_{0}=1$. Now substituting $y_{k}$ from (10) in (1) we have

$$
\begin{gathered}
A(\sigma) y_{k}=A(\sigma)\left[\sum_{i=1}^{q} \sum_{j=1}^{q} H_{-k-i} A_{j} y_{j-i}+\sum_{i=0}^{k+\hat{q}_{r}} \sum_{j=0}^{q} H_{-k+i} B_{j} u_{i+j}\right] \\
=\sum_{n=0}^{q} A_{n} \sum_{i=1}^{q} \sum_{j=1}^{q} H_{-k-i} A_{j} y_{j-i}+\sum_{n=0}^{q} A_{n} \sum_{i=0}^{k+n+\hat{q}_{r}} \sum_{j=0}^{q} H_{-k-n+i} B_{j} u_{i+j} \\
=\sum_{i=1}^{q} \sum_{j=1}^{q} \sum_{n=0}^{q}\left(A_{n} H_{-k-n-i}\right) A_{j} y_{j-i} \\
+\sum_{n=0}^{q} A_{n} H_{-k-n} \sum_{j=0}^{q} B_{j} u_{j}+\sum_{n=0}^{q} A_{n} H_{-k-n+!} \sum_{j=0}^{q} B_{j} u_{j+1}+\cdots+\sum_{n=0}^{q} A_{n} H_{-n} \\
+\sum_{n=0}^{q} A_{n} H_{-n+1} \sum_{j=0}^{q} B_{j} u_{j+k+1} B_{j} u_{j+k} \cdots+\sum_{n=0}^{q} A_{n} H_{-n+\hat{q}_{r}} \sum_{j=0}^{q} B_{j} u_{j+k+\hat{q}_{r}} \\
+\cdots+\sum_{n=0}^{q} A_{n} H_{-n+\hat{q}_{r}+1} \sum_{j=0}^{q} B_{j} u_{j+k+\hat{q}_{r}+1}+\cdots+A_{q} B_{\hat{q}_{r}} \sum_{j=0}^{q} B_{j} u_{j+k+\hat{q}_{r}+q} \\
\stackrel{(11)}{=} \sum_{i=1}^{q} \sum_{j=1}^{q} \delta_{-k-i} A_{j} y_{j-i}+\delta_{-k} \sum_{j=0}^{q} B_{j} u_{j}+\delta_{-k+1} \\
\sum_{j=0}^{q} u_{j+1}+\cdots+\delta_{0} \sum_{j=0} u_{j+k}=B_{(\sigma)} u_{k},
\end{gathered}
$$

which proves the theorem.

It is important to note that the discrete-time ARMA representation does not always have a solution. A necessary and sufficient condition for the ARMA representation (1) to have a solution is that the initial conditions $\left\{y_{0}, y_{1}, \ldots, y_{q-1}\right\}$ satisfy the relation (1) for $k=0,1, \ldots, q-1$. Therefore, we have the following definition. 
Definition 3.2. We define

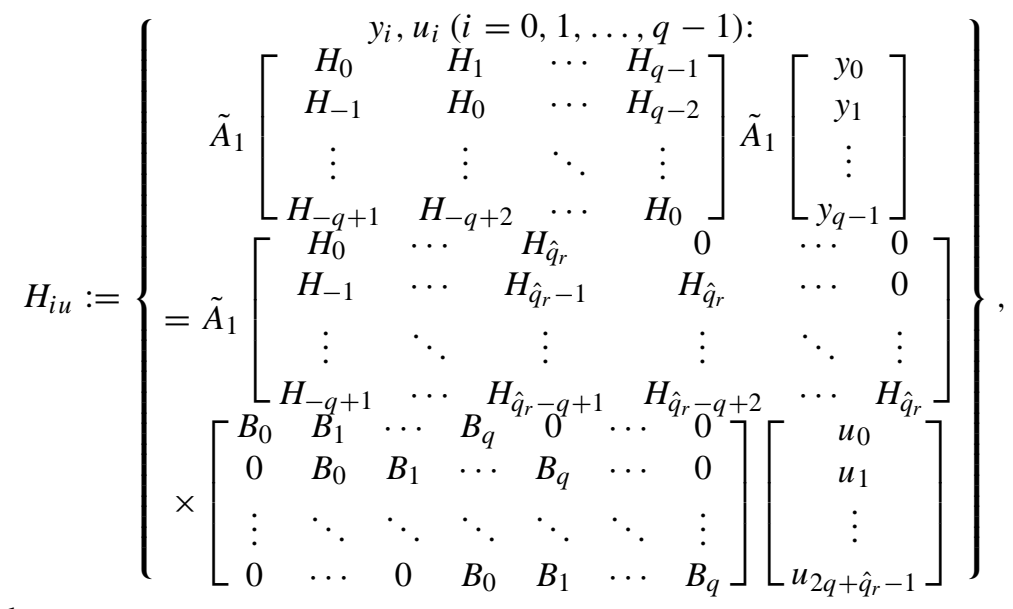

where

$$
\tilde{A}_{1}=\left[\begin{array}{cccc}
A_{0} & 0 & \cdots & 0 \\
A_{1} & A_{0} & \cdots & 0 \\
\vdots & \vdots & \ddots & \vdots \\
A_{q-1} & A_{q-2} & \cdots & A_{0}
\end{array}\right]
$$

as the admissible initial condition space of (1) under nonzero inputs.

Proof. Consider the relation (1) for $k=0,1, \ldots, q-1$ and write this in the form

$$
\begin{aligned}
& {\left[\begin{array}{ccccccc}
A_{q} & A_{q-1} & \cdots & A_{0} & 0 & \cdots & 0 \\
0 & A_{q} & A_{q-1} & \cdots & A_{0} & \cdots & 0 \\
\vdots & \ddots & \ddots & \ddots & \ddots & \ddots & \vdots \\
0 & \cdots & 0 & A_{q} & A_{q-1} & \cdots & A_{0}
\end{array}\right]\left[\begin{array}{c}
y_{2 q-1} \\
y_{2 q-2} \\
\vdots \\
y_{0}
\end{array}\right]} \\
& =\left[\begin{array}{ccccccc}
B_{q} & B_{q-1} & \cdots & B_{0} & 0 & \cdots & 0 \\
0 & B_{q} & B_{q-1} & \cdots & B_{0} & \cdots & 0 \\
\vdots & \ddots & \ddots & \ddots & \ddots & \ddots & \vdots \\
0 & \cdots & 0 & B_{q} & B_{q-1} & \cdots & B_{0}
\end{array}\right]\left[\begin{array}{c}
u_{2 q-1} \\
u_{2 q-2} \\
\vdots \\
u_{0}
\end{array}\right] .
\end{aligned}
$$

Substitution of the values $y_{q}, y_{q+1}, \ldots, y_{2 q-1}$ with the respective formula of (9) and the use of (11) give the result that the initial conditions $\left\{y_{0}, y_{1}, \ldots, y_{q-1}\right\}$ satisfy the system if the relation (13) is satisfied.

As we can see in (9), the solution of (1) is determined in terms of the initial conditions $\left\{y_{0}, y_{1}, \ldots, y_{q-1}\right\}$ and the input sequence of the system. An obvious disadvantage is that for each succesive output $y_{k}$ specified by $k=$ $q, q+1, \ldots$, the coefficient matrices $H_{j}$ comprising each specific solution change. Therefore, if the solution is required over a comparatively large range, say, $y_{q}, y_{q+1}, \ldots, y_{100}$ corresponding to $k=q, q+1, \ldots, 100$, we would require the coefficient matrices $H_{-101}, H_{-100}, \ldots, H_{\hat{q}_{r}}$. An equivalent forward 
solution is presented in what follows for the general solution $y_{k}$ depends on the previous $q$ outputs $\left\{y_{k-1}, y_{k-2}, \ldots, y_{k-q}\right\}$ and not on the $q$ fixed initial conditions $\left\{y_{0}, y_{1}, \ldots, y_{q-1}\right\}$. In this case, the coefficient matrices required over a solution range are fixed (i.e., independent of $k$ ), namely $H_{-q}, H_{-q+1}, \ldots, H_{\hat{q}_{r}}$.

Corollary 3.3. Equation (9) is equivalent to the following forward recursion:

$$
\begin{aligned}
y_{k}= & -\left[\begin{array}{llll}
H_{-1} & H_{-2} & \cdots & H_{-q}
\end{array}\right]\left[\begin{array}{ccccc}
A_{0} & 0 & \cdots & 0 \\
A_{1} & A_{0} & \cdots & 0 \\
\vdots & \vdots & \ddots & \vdots \\
A_{q-1} & A_{q-2} & \cdots & A_{0}
\end{array}\right]\left[\begin{array}{c}
y_{k-1} \\
y_{k-2} \\
\vdots \\
y_{k-q}
\end{array}\right] \\
& +\left[\begin{array}{lllllll}
H_{-q} & H_{-q+1} & \cdots & H_{0} & \cdots & H_{\hat{q}_{r}}
\end{array}\right] \\
& \times\left[\begin{array}{ccccccc}
B_{0} & B_{1} & \cdots & B_{q} & 0 & \cdots & 0 \\
0 & B_{0} & B_{1} & \cdots & B_{q} & \cdots & 0 \\
\vdots & \ddots & \ddots & \ddots & \ddots & \ddots & \vdots \\
0 & \cdots & 0 & B_{0} & B_{1} & \cdots & B_{q}
\end{array}\right]\left[\begin{array}{c}
u_{k-q} \\
u_{k-q+1} \\
\vdots \\
u_{k+\hat{q}_{r}+q}
\end{array}\right]
\end{aligned}
$$

or equivalently

$$
y_{k}=-\sum_{i=1}^{q} \sum_{j=0}^{i-1} H_{-i} A_{j} y_{k-i+j}+\sum_{i=0}^{q+\hat{q}_{r}} \sum_{j=0}^{q} H_{-q+i} B_{j} u_{k-q+j+i} .
$$

Proof. It is easily seen that the state vector $y_{q}$ will be connected with the previous vectors $\left\{y_{0}, y_{1}, \ldots, y_{q-1}\right\}$ according to (9) with the following relation:

$$
\begin{aligned}
y_{q}= & {\left[\begin{array}{llll}
H_{-2 q} & H_{-2 q+1} & \cdots & H_{-q-1}
\end{array}\right]\left[\begin{array}{cccc}
A_{q} & 0 & \cdots & 0 \\
A_{q-1} & A_{q} & \cdots & 0 \\
\vdots & \vdots & \ddots & \vdots \\
A_{1} & A_{2} & \cdots & A_{q}
\end{array}\right]\left[\begin{array}{c}
y_{0} \\
y_{1} \\
\vdots \\
y_{q-1}
\end{array}\right] } \\
& +\left[\begin{array}{lllllll}
H_{-q} & H_{-q+1} & \cdots & H_{0} & \cdots & H_{\hat{q}_{r}}
\end{array}\right] \\
& \times\left[\begin{array}{ccccccc}
B_{0} & B_{1} & \cdots & B_{q} & 0 & \cdots & 0 \\
0 & B_{0} & B_{1} & \cdots & B_{q} & \cdots & 0 \\
\vdots & \ddots & \ddots & \ddots & \ddots & \ddots & \vdots \\
0 & \cdots & 0 & B_{0} & B_{1} & \cdots & B_{q}
\end{array}\right]\left[\begin{array}{ccc}
u_{0} \\
u_{1} \\
\vdots \\
u_{2 q+\hat{q}_{r}}
\end{array}\right] \\
& \stackrel{(11)}{=}-\left[\begin{array}{llll}
H_{-1} & H_{-2} & \cdots & H_{-q}
\end{array}\right]\left[\begin{array}{cccc}
A_{0} & 0 & \cdots & 0 \\
A_{1} & A_{0} & \cdots & 0 \\
\vdots & \vdots & \ddots & \vdots \\
A_{q-1} & A_{q-2} & \cdots & A_{0}
\end{array}\right]\left[\begin{array}{c}
y_{q-1} \\
y_{q-2} \\
\vdots \\
y_{0}
\end{array}\right]
\end{aligned}
$$


N. P. KARAMPETAKIS et al.

$$
\begin{aligned}
& +\left[\begin{array}{llllll}
H_{-q} & H_{-q+1} & \cdots & H_{0} & \cdots & H_{\hat{q}_{r}}
\end{array}\right]\left[\begin{array}{ccccccc}
B_{0} & B_{1} & \cdots & B_{q} & 0 & \cdots & 0 \\
0 & B_{0} & B_{1} & \cdots & B_{q} & \cdots & 0 \\
\vdots & \ddots & \ddots & \ddots & \ddots & \ddots & \vdots \\
0 & \cdots & 0 & B_{0} & B_{1} & \cdots & B_{q}
\end{array}\right] \\
& \times\left[\begin{array}{c}
u_{0} \\
u_{1} \\
\vdots \\
u_{2 q+\hat{q}_{r}}
\end{array}\right] .
\end{aligned}
$$

The system is time invariant, and thus the same relation will connect the output $y_{k}$ with the previous vector's $q$ outputs $\left\{y_{k-1}, y_{k-2}, \ldots, y_{k-q}\right\}$. Thus if we replace $\left\{y_{q}, y_{q-1}, \ldots, y_{0}\right\}$ with $\left\{y_{k}, y_{k-1}, \ldots, y_{k-q}\right\}$, respectively, and $\left\{u_{0}, u_{1}, \ldots, u_{2 q+\hat{q}_{r}}\right\}$ with $\left\{u_{k-q}, u_{k-q+1}, \ldots, u_{k+q+\hat{q}_{r}}\right\}$, respectively, we get the relation $(15)$.

As we have already mentioned, the advantage of the formula (15) is that it depends only on the $q+\hat{q}_{r}+1$ Laurent expansion terms $\left\{H_{-q}, H_{-q+1}, \ldots\right.$,

Author:

OK reference to formula (15) herel
$H_{0}, \ldots, H_{\hat{q}_{r}}$. Formula (15) is very useful when we need to determine $y_{k}$ in the closed interval $k=q, q \overline{+1}, q+2, \ldots$ because we always have to start to compute from $y_{q}, y_{q+1}, \ldots$ in contrast to the solution formula (9), only the $q$ first initial conditions are required for the determination of $y_{k}$. Another advantage of (15) is that the round-off errors for the determination of the $q+\hat{q}_{r}+1$ Laurent expansion terms $\left\{H_{-q}, H_{-q+1}, \ldots, H_{0}, \ldots, H_{\hat{q}_{r}}\right\}$ are less than those for the determination of $\left\{H_{-k}, \ldots, H_{\hat{q}_{r}}\right\}$ in (9).

\subsection{The backward solution of ARMA representations}

Consider the ARMA representation (1). The Laurent series expansion about zero for the resolvent matrix is given in (4). Then we have the following theorem.

Theorem 3.4. The whole response of the system will be

$$
\begin{aligned}
& y_{k}=\left[\begin{array}{llll}
V_{N-k} & V_{N-k-1} & \cdots & V_{N-k-q+1}
\end{array}\right]\left[\begin{array}{cccc}
A_{q} & 0 & \cdots & 0 \\
A_{q-1} & A_{q} & \cdots & 0 \\
\vdots & \vdots & \ddots & \vdots \\
A_{1} & A_{2} & \cdots & A_{q}
\end{array}\right]\left[\begin{array}{c}
y_{N} \\
y_{N-1} \\
\vdots \\
y_{N-q+1}
\end{array}\right] \\
& +\left[\begin{array}{llll}
V_{N-k-q} & V_{N-k-q-1} & \cdots & V_{-\ell}
\end{array}\right] \\
& \times\left[\begin{array}{ccccccc}
B_{q} & B_{q-1} & \cdots & B_{0} & 0 & \cdots & 0 \\
0 & B_{q} & B_{q-1} & \cdots & B_{0} & \cdots & 0 \\
\vdots & \ddots & \ddots & \ddots & \ddots & \ddots & \vdots \\
0 & \cdots & 0 & B_{q} & B_{q-1} & \cdots & B_{0}
\end{array}\right]\left[\begin{array}{c}
u_{N} \\
u_{N-1} \\
\vdots \\
u_{k-\ell}
\end{array}\right]
\end{aligned}
$$


or equivalently

$$
y_{k}=\sum_{i=0}^{q-1} \sum_{j=0}^{i} V_{N-k-i} A_{j} y_{N-i+j}+\sum_{i=0}^{q+k-N-\ell} \sum_{j=0}^{q} V_{N-k-q-i} B_{j} u_{N+j-i-q} .
$$

Proof. Consider the dual ARMA representation (8) of (1)

$$
\tilde{A}(\sigma) \tilde{y}_{k}=\tilde{B}(\sigma) \tilde{u}_{k},
$$

where

$\tilde{A}(\sigma)=A_{0} \sigma^{q}+\cdots+A_{q-1} \sigma+A_{q} \quad$ and $\quad \tilde{B}(\sigma)=B_{0} \sigma^{q}+\cdots+B_{q-1} \sigma+B_{q}$.

Consider also the Laurent expansion at $s=\infty$ from (6). Then from Theorem 3.1 the solution of (20) will be

$$
\begin{aligned}
& \tilde{y}_{k}=\left[\begin{array}{llll}
\tilde{H}_{-k-q} & \tilde{H}_{-k-q+1} & \cdots & \tilde{H}_{-k-1}
\end{array}\right] \\
& \times\left[\begin{array}{cccc}
A_{0} & 0 & \cdots & 0 \\
A_{1} & A_{0} & \cdots & 0 \\
\vdots & \vdots & \ddots & \vdots \\
A_{q-1} & A_{q-2} & \cdots & A_{0}
\end{array}\right]\left[\begin{array}{c}
\tilde{y}_{0} \\
\tilde{y}_{1} \\
\vdots \\
\tilde{y}_{q-1}
\end{array}\right] \\
& +\left[\begin{array}{llllll}
\tilde{H}_{-k} & \tilde{H}_{-k+1} & \cdots & \tilde{H}_{0} & \cdots & \tilde{H}_{f}
\end{array}\right] \\
& \times\left[\begin{array}{ccccccc}
B_{q} & B_{q-1} & \cdots & B_{0} & 0 & \cdots & 0 \\
0 & B_{q} & B_{q-1} & \cdots & B_{0} & \cdots & 0 \\
\vdots & \ddots & \ddots & \ddots & \ddots & \ddots & \vdots \\
0 & \cdots & 0 & B_{q} & B_{q-1} & \cdots & B_{0}
\end{array}\right]\left[\begin{array}{c}
\tilde{u}_{0} \\
\tilde{u}_{1} \\
\vdots \\
\tilde{u}_{k+\hat{q}_{r}+q}
\end{array}\right] \\
& \underset{\substack{(6) \\
V_{-i}=\tilde{H}_{f-\ell+i}+f=\ell}}{q+f}\left[\begin{array}{llll}
V_{k} & V_{k-1} & \cdots & V_{-q+k+1}
\end{array}\right]\left[\begin{array}{cccc}
A_{q} & 0 & \cdots & 0 \\
A_{q-1} & A_{q} & \cdots & 0 \\
\vdots & \vdots & \ddots & \vdots \\
A_{1} & A_{2} & \cdots & A_{q}
\end{array}\right]\left[\begin{array}{c}
\tilde{y}_{0} \\
\tilde{y}_{1} \\
\vdots \\
\tilde{y}_{q-1}
\end{array}\right] \\
& +\left[\begin{array}{llll}
V_{-q+k} & V_{-q+k-1} & \cdots & V_{-\ell}
\end{array}\right] \\
& \times\left[\begin{array}{ccccccc}
B_{q} & B_{q-1} & \cdots & B_{0} & 0 & \cdots & 0 \\
0 & B_{q} & B_{q-1} & \cdots & B_{0} & \cdots & 0 \\
\vdots & \ddots & \ddots & \ddots & \ddots & \ddots & \vdots \\
0 & \cdots & 0 & B_{q} & B_{q-1} & \cdots & B_{0}
\end{array}\right]\left[\begin{array}{c}
\tilde{u}_{0} \\
\tilde{u}_{1} \\
\vdots \\
\tilde{u}_{k+\hat{q}_{r}+q}
\end{array}\right]
\end{aligned}
$$

From Theorem 2.2 we have that the solution $y_{k}$ of (1) for an input $u_{k}$ is given by the solution $\tilde{y}_{N-k}$ of (8) for an input $\tilde{u}_{N-k}$, and the converse is true as well. Thus we can replace the initial conditions $\tilde{y}_{i}, \tilde{u}_{i}$ of the system (8) with the final conditions $y_{N-i}, u_{N-i}$ of the system (1) as well as the solution $\tilde{y}_{N-k}$ of (8) with solution $y_{k}$ of (1), which proves the relation (18).

A necessary and sufficient condition for the ARMA representation (1) to have a solution is that the final conditions $\left\{y_{N}, y_{N-1}, \ldots, y_{N-q+1}\right\}$ satisfy (1) for $k=$ $N, N-1, \ldots, N-q+1$. Therefore, we define the following. 
Definition 3.5. We define

$$
\begin{aligned}
& \bar{H}_{i u}:=\left\{y_{i}, u_{i}(i=N, N-1, \ldots, N-q+1):\right. \\
& \tilde{A}_{2}\left[\begin{array}{ccc}
V_{-q} & \cdots & V_{-2 q+1} \\
V_{-q+1} & \cdots & V_{-2 q+2} \\
\vdots & \ddots & \vdots \\
V_{-1} & \cdots & V_{-q}
\end{array}\right] \tilde{A}_{2}\left[\begin{array}{c}
y_{N} \\
y_{N-1} \\
\vdots \\
y_{N-q+1}
\end{array}\right] \\
& =\tilde{A}_{2}\left[\begin{array}{cccccc}
V_{-q} & \cdots & V_{-\ell} & 0 & \cdots & 0 \\
V_{-q+1} & \cdots & V_{-\ell+1} & V_{-\ell} & \cdots & 0 \\
\vdots & \ddots & \vdots & \vdots & \ddots & \vdots \\
V_{-1} & \cdots & V_{-\ell+q-1} & V_{-\ell+q-2} & \cdots & V_{-\ell}
\end{array}\right] \\
& \left.\left[\begin{array}{cccccc}
B_{0} & \cdots & B_{q} & 0 & \cdots & 0 \\
\vdots & \ddots & \ddots & \ddots & \ddots & \vdots \\
0 & \cdots & B_{0} & B_{1} & \cdots & B_{q}
\end{array}\right]\left[\begin{array}{c}
u_{N} \\
u_{N-1} \\
\vdots \\
u_{N-q-\ell+1}
\end{array}\right]\right\} \text {, }
\end{aligned}
$$

where

$$
\tilde{A}_{2}=\left[\begin{array}{cccc}
A_{q} & 0 & \cdots & 0 \\
A_{q-1} & A_{q} & \cdots & 0 \\
\vdots & \vdots & \ddots & \vdots \\
A_{1} & A_{2} & \cdots & A_{q}
\end{array}\right]
$$

Proof. Consider the relation (1) for $k=N-q, N-q-1, \ldots, N-2 q+1$ and write this in the form

$$
\begin{aligned}
& {\left[\begin{array}{ccccccc}
A_{q} & A_{q-1} & \cdots & A_{0} & 0 & \cdots & 0 \\
0 & A_{q} & A_{q-1} & \cdots & A_{0} & \cdots & 0 \\
\vdots & \ddots & \ddots & \ddots & \ddots & \ddots & \vdots \\
0 & \cdots & 0 & A_{q} & A_{q-1} & \cdots & A_{0}
\end{array}\right]\left[\begin{array}{c}
y_{N} \\
y_{N-1} \\
\vdots \\
y_{N-2 q+1}
\end{array}\right]} \\
& =\left[\begin{array}{ccccccc}
B_{q} & B_{q-1} & \cdots & B_{0} & 0 & \cdots & 0 \\
0 & B_{q} & B_{q-1} & \cdots & B_{0} & \cdots & 0 \\
\vdots & \ddots & \ddots & \ddots & \ddots & \ddots & \vdots \\
0 & \cdots & 0 & B_{q} & B_{q-1} & \cdots & B_{0}
\end{array}\right]\left[\begin{array}{c}
u_{N} \\
u_{N-1} \\
\vdots \\
u_{N-2 q+1}
\end{array}\right] .
\end{aligned}
$$

Substitution of the values $y_{N-q}, y_{N-q-1}, \ldots, y_{N-2 q+1}$ with the respective formula of (18) and use of (11) give us that the final conditions $\left\{y_{N}, y_{N-1}, \ldots, y_{N-q+1}\right\}$ satisfy the system iff the relation (21) is satisfied.

A backward solution formula in terms of the following $q$ terms and the input sequence of the system is provided by the following corollary. 
Corollary 3.6. Equation (18) is equivalent to the backward recursion:

$$
\begin{aligned}
y_{k}= & {\left[\begin{array}{llll}
V_{q} & V_{q-1} & \cdots & V_{1}
\end{array}\right]\left[\begin{array}{ccccc}
A_{0} & 0 & \cdots & 0 \\
A_{1} & A_{0} & \cdots & 0 \\
\vdots & \vdots & \ddots & \vdots \\
A_{q-1} & A_{q-2} & \cdots & A_{0}
\end{array}\right]\left[\begin{array}{c}
y_{k+q} \\
y_{k+q-1} \\
\vdots \\
y_{k+1}
\end{array}\right] } \\
& +\left[\begin{array}{lllllll}
V_{0} & V_{-1} & \cdots & V_{-\ell}
\end{array}\right] \\
& \times\left[\begin{array}{ccccccc}
B_{q} & B_{q-1} & \cdots & B_{0} & 0 & \cdots & 0 \\
0 & B_{q} & B_{q-1} & \cdots & B_{0} & \cdots & 0 \\
\vdots & \ddots & \ddots & \ddots & \ddots & \ddots & \vdots \\
0 & \cdots & 0 & B_{q} & B_{q-1} & \cdots & B_{0}
\end{array}\right]\left[\begin{array}{c}
u_{k+q} \\
u_{k+q-1} \\
\vdots \\
u_{k-\ell}
\end{array}\right]
\end{aligned}
$$

or equivalently

$$
y_{k}=\sum_{i=0}^{q-1} \sum_{j=0}^{i} V_{q-i} A_{j} y_{k+q-i+j}+\sum_{i=0}^{-\ell} \sum_{j=0}^{q} V_{-i} B_{j} u_{k+j-i} .
$$

Proof. Following a method similar to the proof of Corollary 3.3 we obtain the result.

The advantage of formula (23) is that it depends only on the $q+\ell+1$ Laurent expansion terms $\left[V_{q}, V_{q-1}, \ldots V_{-\ell}\right]$ and thus we do not need the continuous computation of the Laurent expansion terms, which gives rise to numerical errors.

\subsection{The symmetric solution}

In this section, we consider (1) as a relation between the output $y_{k}$ and the input $u_{k}$ over an interval $k=0,1, \ldots, N$, where $k$ is not necessarily the time index. Such an interpretation is used in economics and elsewhere [7], [9]. Consider the discrete-time ARMA representation (1) and the Laurent series expansion about infinity for its resolvent matrix in (3). Then

Lemma 3.7. (i) A right inverse of the matrix

$$
A_{N}=\left[\begin{array}{ccccccc}
A_{q} & A_{q-1} & \cdots & A_{0} & 0 & \cdots & 0 \\
0 & A_{q} & A_{q-1} & \cdots & A_{0} & \cdots & 0 \\
\vdots & \ddots & \ddots & \ddots & \ddots & \ddots & \vdots \\
0 & \cdots & 0 & A_{q} & A_{q-1} & \cdots & A_{0}
\end{array}\right] \in R^{(N-q+1) p \times(N+1) \ell}
$$

is the following:

$$
A_{N}^{r}=\left[\begin{array}{cccc}
H_{-q} & H_{-q-1} & \cdots & H_{-N} \\
H_{-q+1} & H_{-q} & \cdots & H_{-N+1} \\
\cdots & \cdots & \ddots & \cdots \\
H_{-q+N} & H_{-q+N-1} & \cdots & H_{0}
\end{array}\right] .
$$


N. P. KARAMPETAKIS et al.

(ii) A left inverse of the matrix

$$
T=\left[\begin{array}{cccc}
A_{0} & 0 & \cdots & 0 \\
A_{1} & A_{0} & \cdots & 0 \\
\vdots & \vdots & \ddots & \vdots \\
A_{q} & A_{q-1} & \cdots & A_{0} \\
0 & A_{q} & \cdots & A_{1} \\
\vdots & \vdots & \ddots & \vdots \\
0 & 0 & \cdots & A_{q}
\end{array}\right] \in R^{(N-q+1) p \times(N-2 q+1) \ell}
$$

is the following:

$$
T^{r}=\left[\begin{array}{cccc}
H_{0} & H_{-1} & \cdots & H_{-N+q} \\
H_{1} & H_{0} & \cdots & H_{-N+q+1} \\
\cdots & \cdots & \ddots & \cdots \\
H_{N-2 q} & H_{N-2 q-1} & \cdots & H_{-q}
\end{array}\right] .
$$

Proof. Using the relation (11), we can easily show that $A_{N} \times A_{N}^{r}=I$ and $T^{r} \times$ $T=I$, which proves the Lemma.

We can now shoe the following.

Theorem 3.8. The solution of the ARMA representation (1) in terms of the initial and final conditions $\left\{y_{0}, y_{1}, \ldots, y_{q-1}\right\}$ and $\left\{y_{N}, y_{N-1}, \ldots, y_{N-q+1}\right\}$, respectively, is given by the following formula:

$$
\begin{aligned}
& y_{k}=\left[\begin{array}{llll}
H_{-k-1} & H_{-k-2} & \cdots & H_{-k-q}
\end{array}\right]\left[\begin{array}{cccc}
A_{q} & A_{q-1} & \cdots & A_{1} \\
0 & A_{q} & \cdots & A_{2} \\
\vdots & \vdots & \ddots & \vdots \\
0 & 0 & \cdots & A_{q}
\end{array}\right]\left[\begin{array}{c}
y_{q-1} \\
y_{q-2} \\
\vdots \\
y_{0}
\end{array}\right] \\
& +\left[\begin{array}{llll}
H_{N-k} & H_{N-k-1} & \cdots & H_{N-k-q+1}
\end{array}\right]\left[\begin{array}{cccc}
A_{0} & 0 & \cdots & 0 \\
A_{1} & A_{0} & \cdots & 0 \\
\vdots & \vdots & \ddots & \vdots \\
A_{q-1} & A_{q-2} & \cdots & A_{0}
\end{array}\right]\left[\begin{array}{c}
y_{N} \\
y_{N-1} \\
\vdots \\
y_{N-q+1}
\end{array}\right] \\
& +\left[\begin{array}{llll}
H_{N-k-q} & H_{N-k-q-1} & \cdots & H_{-k}
\end{array}\right]\left[\begin{array}{ccccccc}
B_{q} & B_{q-1} & \cdots & B_{0} & 0 & \cdots & 0 \\
0 & B_{q} & B_{q-1} & \cdots & B_{0} & \cdots & 0 \\
\vdots & \ddots & \ddots & \ddots & \ddots & \ddots & \vdots \\
0 & \cdots & 0 & B_{q} & B_{q-1} & \cdots & B_{0}
\end{array}\right]\left[\begin{array}{c}
u_{N} \\
u_{N-1} \\
\vdots \\
u_{0}
\end{array}\right]
\end{aligned}
$$

or equivalently

$$
\begin{aligned}
y_{k}=\sum_{i=1}^{q} & \sum_{j=1}^{q} H_{-k-i} A_{j} y_{j-i}+\sum_{i=0}^{q-1} \sum_{j=0}^{i} H_{N-k-i} A_{j} y_{N-i+j} \\
& +\sum_{i=0}^{N-q} \sum_{j=0}^{q} H_{N-k-q-i} B_{j} u_{N+j-i-q}
\end{aligned}
$$


under the following restrictions between the initial conditions, final conditions, and input sequences:

$$
\left[\begin{array}{ll}
W_{11} & W_{12} \\
W_{21} & W_{22}
\end{array}\right]\left[\begin{array}{c}
X_{A} y_{N-q+1, N} \\
X_{\tilde{A}} y_{0, q-1}
\end{array}\right]=\left[\begin{array}{l}
Z_{1} \\
Z_{2}
\end{array}\right] B_{N} u_{0, N},
$$

where

$$
\begin{aligned}
& W_{11}=\left[\begin{array}{cccc}
H_{-q} & H_{-q-1} & \cdots & H_{-2 q+1} \\
H_{-q+1} & H_{-q} & \cdots & H_{-2 q+2} \\
\vdots & \vdots & \ddots & \vdots \\
H_{-1} & H_{-2} & \cdots & H_{-q}
\end{array}\right] \\
& W_{12}=\left[\begin{array}{cccc}
H_{-N+q-1} & H_{-N+q-2} & \cdots & H_{-N} \\
H_{-N+q} & H_{-N+q-1} & \cdots & H_{-N+1} \\
\vdots & \vdots & \ddots & \vdots \\
H_{-N+2 q-2} & H_{-N+2 q-3} & \cdots & H_{-N+q-1}
\end{array}\right] \\
& W_{21}=\left[\begin{array}{cccc}
H_{N-2 q+1} & H_{N-2 q} & \cdots & H_{N-3 q+2} \\
H_{N-2 q+2} & H_{N-2 q+1} & \cdots & H_{N-3 q+3} \\
\vdots & \vdots & \ddots & \vdots \\
H_{N-q} & H_{N-q-1} & \cdots & H_{N-2 q+1}
\end{array}\right] \\
& W_{22}=\left[\begin{array}{cccc}
H_{0} & H_{-1} & \cdots & H_{-q+1} \\
H_{1} & H_{0} & \cdots & H_{-q+2} \\
\vdots & \vdots & \ddots & \vdots \\
H_{q-1} & H_{q-2} & \cdots & H_{0}
\end{array}\right] \\
& X_{A}=\left[\begin{array}{cccc}
A_{q} & A_{q-1} & \cdots & A_{1} \\
0 & A_{q} & \cdots & A_{2} \\
\vdots & \vdots & \ddots & \vdots \\
0 & 0 & \cdots & A_{q}
\end{array}\right] \\
& X_{\tilde{A}}=\left[\begin{array}{cccc}
A_{0} & 0 & \cdots & 0 \\
A_{1} & A_{0} & \cdots & 0 \\
\vdots & \vdots & \ddots & \vdots \\
A_{q-1} & A_{q-2} & \cdots & A_{0}
\end{array}\right] \\
& Y_{N-q+1, N}=\left[\begin{array}{c}
y_{N} \\
y_{N-1} \\
\vdots \\
y_{N-q+1}
\end{array}\right] ; \quad y_{0, q-1}=\left[\begin{array}{c}
y_{q_{1}} \\
y_{q_{2}} \\
\vdots \\
y_{0}
\end{array}\right] ; \quad u_{0, N}=\left[\begin{array}{c}
u_{N} \\
u_{N-1} \\
\vdots \\
u_{0}
\end{array}\right] \\
& B_{N}=\left[\begin{array}{ccccccc}
B_{0} & B_{1} & \cdots & B_{q} & 0 & \cdots & 0 \\
0 & B_{0} & \cdots & B_{q-1} & B_{q} & \cdots & 0 \\
\vdots & \vdots & \ddots & \ddots & \ddots & \ddots & \vdots \\
0 & 0 & \cdots & B_{0} & B_{1} & \cdots & B_{q}
\end{array}\right]
\end{aligned}
$$


N. P. Karampetakis et al.

$$
\begin{aligned}
& Z_{1}= {\left[\begin{array}{cccc}
H_{-q} & H_{-q-1} & \cdots & H_{-N} \\
H_{-q+1} & H_{-q} & \cdots & H_{-N+1} \\
\vdots & \vdots & \ddots & \vdots \\
H_{-1} & H_{-2} & \cdots & H_{-N+q-1}
\end{array}\right] ; } \\
& Z_{2}=\left[\begin{array}{cccc}
H_{N-2 q+1} & H_{N-2 q} & \cdots & H_{-q+1} \\
H_{N-2 q+2} & H_{N-2 q+1} & \cdots & H_{-q+2} \\
\vdots & \vdots & \ddots & \vdots \\
H_{N-q} & H_{N-q-1} & \cdots & H_{0}
\end{array}\right] .
\end{aligned}
$$

We call solution (25) the symmetric solution of (1) and equations (27) boundary mapping equations of (1).

Proof. We rewrite (1) in the form

$$
\begin{aligned}
& \underbrace{\left[\begin{array}{cccccc}
A_{q} & \cdots & A_{0} & 0 & \cdots & 0 \\
\vdots & \ddots & \ddots & \ddots & \ddots & \vdots \\
0 & \cdots & A_{q} & A_{q-1} & \cdots & A_{0}
\end{array}\right]}_{A_{N}} \underbrace{\left[\begin{array}{c}
y_{N} \\
y_{N-1} \\
\vdots \\
y_{0}
\end{array}\right]}_{y_{0, N}} \\
& =\underbrace{\left[\begin{array}{cccccc}
B_{q} & \cdots & B_{0} & 0 & \cdots & 0 \\
\vdots & \ddots & \ddots & \ddots & \ddots & \vdots \\
0 & \cdots & B_{q} & B_{q-1} & \cdots & B_{0}
\end{array}\right]}_{B_{N}} \underbrace{\left[\begin{array}{c}
u_{N} \\
u_{N-1} \\
\vdots \\
u_{0}
\end{array}\right]}_{u_{0, N}} \\
& \Leftrightarrow\left[\begin{array}{c}
X_{A} y_{N-q+1, N} \\
0 \\
X_{\tilde{A}} y_{0, q-1}
\end{array}\right]=\left[\begin{array}{cccc}
-A_{0} & \cdots & 0 \\
\vdots & \ddots & \vdots \\
-A_{q} & \ddots & -A_{0} & \mid B_{N} \\
\vdots & \ddots & \vdots \\
0 & \cdots & -A_{q}
\end{array}\right]\left[\begin{array}{c}
y_{q, N-q} \\
u_{0, N}
\end{array}\right],
\end{aligned}
$$

where $y_{q, N-q}=\left[y_{N-q}^{T}, \ldots, y_{q}^{T}\right]^{T}$. Premultiplying both sides of (29) by $A_{N}^{r}$, we obtain from the first $q$ and the last $q$ equations the relations (27), while from the middle $N-2 q$ equations, after the use of Lemma 3.7, we obtain formula (25).

A necessary and sufficient condition so that the ARMA representation (1) has a solution is that the initial, and final conditions and input sequences satisfy relation (27). Therefore we have the following definition. 
Definition 3.9. We define as

$$
\begin{gathered}
\tilde{H}_{i u}:=\left\{y_{0, q-1}, y_{N-q+1, N}:\right. \\
\left.\left[\begin{array}{ll}
W_{11} & W_{12} \\
W_{21} & W_{22}
\end{array}\right]\left[\begin{array}{c}
X_{A} y_{N-q+1, N} \\
X_{\tilde{A}} y_{0, q-1}
\end{array}\right]=\left[\begin{array}{c}
Z_{1} \\
Z_{2}
\end{array}\right] B_{N} u_{0, N}\right\}
\end{gathered}
$$

the symmetric boundary condition space of (1) under nonzero inputs.

The boundary mapping equation (27) represents the restrictions that the system places on the boundary variables $y_{0, q-1}, y_{N-q+1, N}$ in order for the system to be solvable. Additional restrictions on the variables can be applied to the system in the form of an auxiliary equation

$$
W_{31} y_{N-q+1, N}+W_{32} y_{0, q-1}=C .
$$

The combined boundary equation formed from (27) and (31)

$$
\begin{gathered}
{\left[\begin{array}{cc}
W_{11} X_{A} & W_{12} X_{\tilde{A}} \\
W_{21} X_{A} & W_{22} X_{\tilde{A}} \\
W_{31} & W_{32}
\end{array}\right]\left[\begin{array}{c}
y_{N-q+1, N} \\
y_{0, q-1}
\end{array}\right]=\left[\begin{array}{c}
Z_{1} u_{0, N} \\
Z_{2} u_{0, N} \\
C
\end{array}\right]} \\
\Leftrightarrow Z Y=\tilde{C}
\end{gathered}
$$

will subsequently define a unique solution iff $Z Z^{+} \tilde{C}=\tilde{C}$ and $Z$ has full column rank, where $Z^{+}$denotes the pseudoinverse of $Z$, i.e., $Y=Z^{+} \tilde{C}$.

Alternative forms of the solution formula (25) are given by the following corollary.

Corollary 3.10. The symmetric solution (25) can be written in the alternative forms:

Forward-symmetric

$$
\begin{aligned}
& y_{k}=-\left[\begin{array}{llll}
H_{-1} & H_{-2} & \cdots & H_{-q}
\end{array}\right]\left[\begin{array}{cccc}
A_{0} & 0 & \cdots & 0 \\
A_{1} & A_{0} & \cdots & 0 \\
\vdots & \vdots & \ddots & \vdots \\
A_{q-1} & A_{q-2} & \cdots & A_{0}
\end{array}\right]\left[\begin{array}{c}
y_{k-1} \\
y_{k-2} \\
\vdots \\
y_{k-q}
\end{array}\right] \\
& +\left[\begin{array}{llll}
H_{N-k} & H_{N-k-1} & \cdots & H_{N-k-q+1}
\end{array}\right] \\
& \times\left[\begin{array}{cccc}
A_{0} & 0 & \cdots & 0 \\
A_{1} & A_{0} & \cdots & 0 \\
\vdots & \vdots & \ddots & \vdots \\
A_{q-1} & A_{q-2} & \cdots & A_{0}
\end{array}\right]\left[\begin{array}{c}
y_{N} \\
y_{N-1} \\
\vdots \\
y_{N-q+1}
\end{array}\right] \\
& +\left[\begin{array}{llll}
H_{N-k-q} & H_{N-k-q-1} & \cdots & H_{-k}
\end{array}\right] \\
& \times\left[\begin{array}{ccccccc}
B_{q} & B_{q-1} & \cdots & B_{0} & 0 & \cdots & 0 \\
0 & B_{q} & B_{q-1} & \cdots & B_{0} & \cdots & 0 \\
\vdots & \ddots & \ddots & \ddots & \ddots & \ddots & \vdots \\
0 & \cdots & 0 & B_{q} & B_{q-1} & \cdots & B_{0}
\end{array}\right]\left[\begin{array}{c}
u_{N} \\
u_{N-1} \\
\vdots \\
u_{0}
\end{array}\right]
\end{aligned}
$$


or

$$
\begin{gathered}
y_{k}=\sum_{i=1}^{q} \sum_{j=0}^{i-1} H_{-i} A_{j} y_{k-j-i}+\sum_{i=0}^{q-1} \sum_{j=0}^{i} H_{N-k-i} A_{j} y_{N-i+j} \\
+\sum_{i=0}^{N-k} \sum_{j=0}^{q} H_{N-k-q-i} B_{j} u_{N+j-i-q}
\end{gathered}
$$

Backward-symmetric

$$
\begin{aligned}
& y_{k}=\left[\begin{array}{llll}
H_{-k-1} & H_{-k-2} & \cdots & H_{-k-q}
\end{array}\right]\left[\begin{array}{cccc}
A_{q} & A_{q-1} & \cdots & A_{1} \\
0 & A_{q} & \cdots & A_{2} \\
\vdots & \vdots & \ddots & \vdots \\
0 & 0 & \cdots & A_{q}
\end{array}\right]\left[\begin{array}{c}
y_{q-1} \\
y_{q-2} \\
\vdots \\
y_{0}
\end{array}\right] \\
& -\left[\begin{array}{llll}
H_{0} & H_{-1} & \cdots & H_{-q+1}
\end{array}\right]\left[\begin{array}{cccc}
A_{q} & A_{q-1} & \cdots & A_{1} \\
0 & A_{q} & \cdots & A_{2} \\
\vdots & \vdots & \ddots & \vdots \\
0 & 0 & \cdots & A_{q}
\end{array}\right]\left[\begin{array}{c}
y_{k+q} \\
y_{k+q-1} \\
\vdots \\
y_{k+1}
\end{array}\right] \\
& +\left[\begin{array}{llll}
H_{0} & H_{-1} & \cdots & H_{-k}
\end{array}\right]\left[\begin{array}{ccccccc}
B_{q} & B_{q-1} & \cdots & B_{0} & 0 & \cdots & 0 \\
0 & B_{q} & B_{q-1} & \cdots & B_{0} & \cdots & 0 \\
\vdots & \ddots & \ddots & \ddots & \ddots & \ddots & \vdots \\
0 & \cdots & 0 & B_{q} & B_{q-1} & \cdots & B_{0}
\end{array}\right] \\
& \times\left[\begin{array}{c}
u_{k+q} \\
u_{k+q-1} \\
\vdots \\
u_{0}
\end{array}\right]
\end{aligned}
$$

or equivalently

$$
y_{k}=\sum_{i=1}^{q} \sum_{j=i}^{q} H_{-k-i} A_{j} y_{j-i}-\sum_{i=0}^{q-1} \sum_{j=i+1}^{q} H_{-i} A_{j} y_{k+j-i}+\sum_{i=0}^{k} \sum_{j=0}^{q} H_{-i} B_{j} u_{k+j-i}
$$

Proof. Taking the solution formula (25), we use the following three tasks.

(i) Assume that $k=v q+v(N-k=v q+v)$. 
(ii) Make the following replacement:

$$
\begin{aligned}
& {\left[\begin{array}{llll}
H_{-s} & H_{-s+1} & \cdots & H_{-s+q-1}
\end{array}\right]\left[\begin{array}{cccc}
A_{q} & A_{q-1} & \cdots & A_{1} \\
0 & A_{q} & \cdots & A_{2} \\
\vdots & \vdots & \ddots & \vdots \\
0 & 0 & \cdots & A_{q}
\end{array}\right]} \\
& \stackrel{\text { for } s \neq q}{=}-\left[\begin{array}{llll}
H_{-s+q} & H_{-s+q+1} & \cdots & H_{-s+2 q-1}
\end{array}\right] \\
& \times\left[\begin{array}{cccc}
A_{0} & A_{1} & \cdots & A_{q-1} \\
0 & A_{0} & \cdots & A_{q-2} \\
\vdots & \vdots & \ddots & \vdots \\
0 & 0 & \cdots & A_{0}
\end{array}\right] \\
& =\left[\begin{array}{llll}
H_{N-k-s q} & H_{N-k-s q-1} & \cdots & H_{N-k-(s+1) q+1}
\end{array}\right] \\
& \times\left[\begin{array}{cccc}
A_{0} & 0 & \cdots & 0 \\
A_{1} & A_{0} & \cdots & 0 \\
\vdots & \vdots & \ddots & \vdots \\
A_{q-1} & A_{q-2} & \cdots & A_{0}
\end{array}\right] \\
& \stackrel{\text { for }_{s} \neq q}{=}-\left[\begin{array}{llll}
H_{N-k-(s+1) q} & H_{N-k-(s+1) q-1} & \cdots & H_{N-k-(s+2) q+1}
\end{array}\right] \\
& \times\left[\begin{array}{cccc}
A_{q} & A_{q-1} & \cdots & A_{1} \\
0 & A_{q} & \cdots & A_{2} \\
\vdots & \vdots & \ddots & \vdots \\
0 & 0 & \cdots & A_{q}
\end{array}\right]
\end{aligned}
$$

which is based on (11).

(iii) Make the following replacement (using (1)):

$$
\begin{aligned}
& {\left[\begin{array}{cccc}
A_{0} & A_{1} & \cdots & A_{q-1} \\
0 & A_{0} & \cdots & A_{q-2} \\
\vdots & \vdots & \ddots & \vdots \\
0 & 0 & \cdots & A_{0}
\end{array}\right]\left[\begin{array}{c}
y_{k+s q} \\
y_{k+s q+1} \\
\vdots \\
y_{k+(s+1) q-1}
\end{array}\right]} \\
& =-\left[\begin{array}{cccc}
A_{q} & 0 & \cdots & 0 \\
A_{q-1} & A_{q} & \cdots & 0 \\
\vdots & \vdots & \ddots & \vdots \\
A_{1} & A_{2} & \cdots & A_{q}
\end{array}\right]\left[\begin{array}{c}
y_{k+(s+1) q} \\
y_{k+(s+1) q+1} \\
\vdots \\
y_{k+(s+2) q-1}
\end{array}\right] \\
& +\left[\begin{array}{ccccccc}
B_{0} & B_{1} & \cdots & B_{q} & 0 & \cdots & 0 \\
0 & B_{0} & \cdots & B_{q-1} & B_{q} & \cdots & 0 \\
\vdots & \vdots & \ddots & \ddots & \ddots & \ddots & \vdots \\
0 & 0 & \cdots & B_{0} & B_{1} & \cdots & B_{q}
\end{array}\right]\left[\begin{array}{c}
u_{k+s q} \\
u_{k+s q+1} \\
\vdots \\
u_{k+(s+2) q-1}
\end{array}\right]
\end{aligned}
$$


N. P. KARAMPETAKIS et al.

$$
\begin{aligned}
& {\left[\begin{array}{cccc}
A_{q} & A_{q-1} & \cdots & A_{1} \\
0 & A_{q} & \cdots & A_{2} \\
\vdots & \vdots & \ddots & \vdots \\
0 & 0 & \cdots & A_{q}
\end{array}\right]\left[\begin{array}{c}
y_{N-s q} \\
y_{N-s q-1} \\
\vdots \\
y_{N-(s+1) q+1}
\end{array}\right]} \\
& =-\left[\begin{array}{cccc}
A_{0} & 0 & \cdots & 0 \\
A_{1} & A_{0} & \cdots & 0 \\
\vdots & \vdots & \ddots & \vdots \\
A_{q-1} & A_{q-2} & \cdots & A_{0}
\end{array}\right]\left[\begin{array}{c}
y_{N-(s+1) q} \\
y_{N-(s+1) q-1} \\
\vdots \\
y_{N-(s+2) q+1}
\end{array}\right] \\
& +\left[\begin{array}{ccccccc}
B_{0} & B_{1} & \cdots & B_{q} & 0 & \cdots & 0 \\
0 & B_{0} & \cdots & B_{q-1} & B_{q} & \cdots & 0 \\
\vdots & \vdots & \ddots & \ddots & \ddots & \ddots & \vdots \\
0 & 0 & \cdots & B_{0} & B_{1} & \cdots & B_{q}
\end{array}\right]\left[\begin{array}{c}
u_{N-s q} \\
u_{N-s q-1} \\
\vdots \\
u_{N-(s+2) q+1}
\end{array}\right]
\end{aligned}
$$

which is based on (1). We now obtain the solution formulas (33) and (35).

In the forward-symmetric case, we still solve within the region $[0, N]$, but now the solution depends on the $q$ final conditions $\left\{y_{N}, y_{N-1}, \ldots, y_{N-q+1}\right\}$ and the previous $q$ outputs $\left\{y_{k-1}, y_{k-2}, \ldots, y_{k-q}\right\}$ and no longer on the $q$ fixed initial conditions $\left\{y_{0}, y_{1}, \ldots, y_{q-1}\right\}$. Therefore, we solve forward in the interval.

In the backward-symmetric case, we again still solve within the region $[0, N]$, but now the solution depends on the $q$ initial conditions $\left\{y_{0}, y_{1}, \ldots, y_{q-1}\right\}$ and the future $q$ outputs $\left\{y_{k+1}, y_{k+2}, \ldots, y_{k+q}\right\}$ and no longer on the $q$ fixed final conditions $\left\{y_{N}, y_{N-1}, \ldots, y_{N-q+1}\right\}$. Therefore, we solve backward in the interval.

\section{Illustrative example}

Consider the following discrete-time ARMA representation:

$$
\left[\begin{array}{ccc}
\sigma^{2}+5 \sigma+6 & \sigma+1 & 0 \\
2 \sigma-5 & 3 \sigma+2 & 1 \\
0 & -1 & 0
\end{array}\right]\left[\begin{array}{l}
y_{k}^{1} \\
y_{k}^{2} \\
y_{k}^{3}
\end{array}\right]=\underset{B(\sigma)=B_{0}}{\left[\begin{array}{l}
0 \\
0 \\
y_{k}
\end{array}\right]} u_{k} .
$$

Consider also the Laurent expansion of $A(\sigma)^{-1}$ at $s=\infty$

$$
\begin{aligned}
A(\sigma)^{-1}= & {\left[\begin{array}{lll}
0 & 0 & 0 \\
0 & 0 & 0 \\
0 & 0 & 3
\end{array}\right] \sigma+\left[\begin{array}{rrr}
0 & 0 & 0 \\
0 & 0 & -1 \\
0 & 1 & 0
\end{array}\right]+\left[\begin{array}{rrr}
0 & 0 & 1 \\
0 & 0 & 0 \\
-2 & 0 & 13
\end{array}\right] \sigma^{-1} } \\
& +\left[\begin{array}{rrr}
1 & 0 & -4 \\
0 & 0 & 0 \\
15 & 0 & -48
\end{array}\right] \sigma^{-2}+\left[\begin{array}{rrr}
-5 & 0 & 14 \\
0 & 0 & 0 \\
-63 & 0 & 162
\end{array}\right] \sigma^{-3}+\cdots \\
H_{-2} & H_{-3}
\end{aligned}
$$


and the Laurent expansion at $s=0$ of $A(\sigma)^{-1}$

$$
\begin{aligned}
A(\sigma)^{-1}= & {\left[\begin{array}{ccc}
\frac{1}{6} & 0 & \frac{1}{6} \\
0 & 0 & -1 \\
\frac{5}{6} & 1 & \frac{17}{6}
\end{array}\right]+\left[\begin{array}{ccc}
-\frac{5}{36} & 0 & \frac{1}{36} \\
0 & 0 & 0 \\
-\frac{37}{36} & 0 & \frac{101}{36}
\end{array}\right] \sigma } \\
& +\left[\begin{array}{ccc}
\frac{19}{216} & 0 & -\frac{11}{216} \\
0 & 0 & 0 \\
\frac{155}{216} & 0 & -\frac{67}{216}
\end{array}\right] \sigma^{2}+\cdots
\end{aligned}
$$

A forward recursive representation of (41) is given according to Corollary 3.3 by

$$
\begin{aligned}
y_{k}= & -\left[\begin{array}{ll}
H_{-1} & H_{-2}
\end{array}\right]\left[\begin{array}{cc}
A_{0} & 0 \\
A_{1} & A_{0}
\end{array}\right]\left[\begin{array}{l}
y_{k-1} \\
y_{k-2}
\end{array}\right] \\
& +\left[\begin{array}{llll}
H_{-2} & H_{-1} & H_{0} & H_{1}
\end{array}\right]\left[\begin{array}{cccc}
B_{0} & 0 & 0 & 0 \\
0 & B_{0} & 0 & 0 \\
0 & 0 & B_{0} & 0 \\
0 & 0 & 0 & B_{0}
\end{array}\right]\left[\begin{array}{c}
u_{k-2} \\
u_{k-1} \\
u_{k} \\
u_{k+1}
\end{array}\right] \\
& =\left[\begin{array}{c}
-5 y_{k-1}^{1}-6_{k-2}^{1}-5 y_{k-2}^{2}-4 u_{k-2}+u_{k-1} \\
-63 y_{k-1}^{1}-90 y_{k-2}^{1}-63 y_{k-2}^{2}-48 u_{k-2}+13 u_{k-1}+3 u_{k+1}
\end{array}\right] .
\end{aligned}
$$

The admissible initial condition space $H_{i u}$ of (41) under nonzero inputs is given from (13) as follows:

$$
H_{\text {iu }}:=\left\{\begin{array}{c}
{\left[\begin{array}{cc}
A_{0} & 0 \\
A_{1} & A_{0}
\end{array}\right]\left[\begin{array}{cc}
H_{0}, u_{i}(i=0,1): \\
H_{-1} & H_{0}
\end{array}\right]\left[\begin{array}{cc}
A_{0} & 0 \\
A_{1} & A_{0}
\end{array}\right]\left[\begin{array}{c}
y_{0} \\
y_{1}
\end{array}\right]} \\
=\left[\begin{array}{cc}
A_{0} & 0 \\
A_{1} & A_{0}
\end{array}\right]\left[\begin{array}{ccc}
H_{0} & H_{1} & 0 \\
H_{-1} & H_{0} & H_{1}
\end{array}\right]\left[\begin{array}{ccc}
B_{0} & 0 & 0 \\
0 & B_{0} & 0 \\
0 & 0 & B_{0}
\end{array}\right]\left[\begin{array}{l}
u_{0} \\
u_{1} \\
u_{2}
\end{array}\right]
\end{array}\right\}
$$

or equivalently

$$
H_{i u}:=\left\{\left[\begin{array}{rrrrrr}
0 & -4 & 0 & 0 & 1 & 0 \\
-5 & 2 & 1 & 2 & 3 & 0 \\
0 & -1 & 0 & 0 & 0 & 0 \\
0 & -6 & 0 & 0 & 1 & 0 \\
-12 & -10 & 0 & -15 & 2 & 1 \\
0 & 0 & 0 & 0 & -1 & 0
\end{array}\right]\left[\begin{array}{l}
y_{0}^{1} \\
y_{0}^{2} \\
y_{0}^{3} \\
y_{1}^{1} \\
y_{1}^{2} \\
y_{1}^{3}
\end{array}\right]-\left[\begin{array}{rrr}
4 & -1 & 0 \\
0 & 0 & 0 \\
1 & 0 & 0 \\
6 & -1 & 0 \\
8 & -2 & 3 \\
0 & 1 & 0
\end{array}\right]\left[\begin{array}{c}
u_{0} \\
u_{1} \\
u_{2}
\end{array}\right]\right\} .
$$

A backward recursive representation of (41) is given from Corollary 3.6 by

$$
\begin{aligned}
y_{k}= & {\left[\begin{array}{ll}
V_{2} & V_{1}
\end{array}\right]\left[\begin{array}{cc}
A_{0} & 0 \\
A_{1} & A_{0}
\end{array}\right]\left[\begin{array}{l}
y_{k+2} \\
y_{k+1}
\end{array}\right]+V_{0} B_{0} u_{k} } \\
= & {\left[\begin{array}{c}
-\frac{1}{6} y_{k+2}^{1}-\frac{5}{6} y_{k+1}^{1}-\frac{1}{6} y_{k+1}^{2}+\frac{1}{6} u_{k} \\
-\frac{5}{6} y_{k+2}^{1}-\frac{37}{6} y_{k+1}^{1}-\frac{25}{6} y_{k+1}^{2}+\frac{17}{6} u_{k}
\end{array}\right] . }
\end{aligned}
$$


The admissible final condition space $\tilde{H}_{i u}$ of (41) under nonzero inputs is given by (21) as follows:

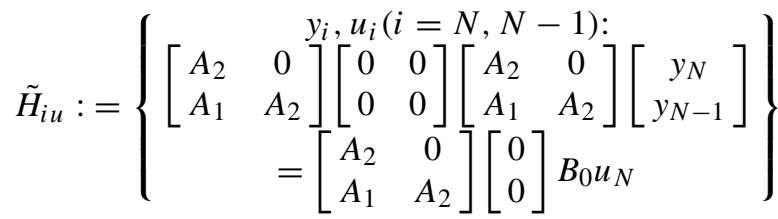

$$
\begin{aligned}
& \equiv\left\{y_{i}, u_{i}(i=N, N-1) \text { : arbitrary }\right\} .
\end{aligned}
$$

In the same way, we can use relation (25) to find the symmetric solution of (41) under the restrictions between the final and initial conditions described by (27).

\section{Conclusions}

In the case of regular discrete-time ARMA representations, exact solutions where proposed in three different forms: (a) forward solutions, (b) backward solutions, and (c) symmetric solutions. It is easily seen that the proposed solutions are extensions of the ones proposed in [8] for discrete-time generalized state space systems. The solution formula presented in this work was implemented via MAPLE in a recent publication [4]. Certain controllability, reachability, and observability criteria based on the proposed solutions are being studied and will be discussed in a future publication.

\section{References}

[1] S. L. Campbell, Singular Systems of Differential Equations, Pitman, San Francisco, CA, 1980.

[2] N. Declaris and A. Rindos, Semistate analysis of neural networks, in Apysia Californica, Proc. 27th MSCS, pp. 686-689, 1984.

[3] G. Fragulis, B. G. Mertzios, and A. I. Vardulakis, Computation of the inverse of a polynomial matrix and evaluation of its Laurent expansion, Int. J. Control, 53, 431-443, 1991.

[4] J. Jones, N. Karampetakis, and A. C. Pugh, Solution of discrete ARMA representations via MAPLE, Proceedings of the European Control Conference, 1997.

[5] N. Karampetakis, J. Jones, and A. C. Pugh, Solution of an ARMA representation via its boundary mapping equation, MTNS 96, 1996.

[6] F. L. Lewis, Fundamental, reachability and observability matrices for descriptor systems, IEEE Trans. Automat. Control, AC-30, 502-505, 1985.

[7] F. L. Lewis, A survey of linear singular systems, Circuit Systems Signal Process, 5, 3-36, 1986.

[8] F. L. Lewis and B. G. Mertzios, On the analysis of discrete linear time-invariant singular systems, IEEE Trans. Automat. Control, 35, 506-511, 1990.

[9] D. G. Luenberger, Dynamic equations in descriptor form, IEEE Trans. Automat. Control, vol. AC-22, 312-321, 1977.

[10] D. G. Luenberger, Time-invariant descriptor systems, Automatica, vol. 14, 473-480, 1978.

[11] B. G. Mertzios and F. L. Lewis, Fundamental matrix of discrete singular systems, Circuits Systems Signal Process, vol. 8, no. 3, 341-355, 1989. 
[12] R. W. Newcobb, The semistate description of nonlinear time-variable circuits, IEEE Trans. Circuits and Systems, vol. 28, 62-71, 1981.

[13] R. Nikoukhah, A. S. Willsky, and B. Levy, Boundary-value descriptor systems: Well posedness, reachability, and observability, Int. J. Control, 46, 1715-1737, 1987.

[14] B. Stoot, Power system dynamic response calculations, Proc. IEEE, 67, 219-247, 1979.

[15] J. H. Wilkinson, Linear differential equations and Kronecker's canonical form, in Recent Advances in Numerical Analysis, edited by C. de Boor and G. Golub, Academic Press, New York, pp. 231-265, 1978.

[16] J. C. Willems, Paradigms and puzzles in the theory of dynamical systems, IEEE Trans. Automat. Control, AC-36, 259-294, 1991. 